\title{
The Model of Hysteretic Behavior of SMA Based on the High Order Approximation of Differential Equation Method
}

\author{
A.A. Likhachev and Yu.N. Koval \\ Institute of Metal Physics of N. A. Sci. of Ukraine, 36 Vernadsky Str., Kiev 252142, Ukraine
}

\begin{abstract}
Due to thermomechanical hysteresis in solids undergoing martensitic type of structure transformations the macroscopic strain and volume fraction of martensite are not a single-valued function of stress and temperature, but they become functions of the process of their change. Some of the phenomenological approaches describing main thermomechanical properties of shape-memory alloys (SMA) connected with hysteresis were recently developed. A special type of differential equations (DE) describing evolution of the inelastic macroscopic strain and volume fraction of martensite as functions of the temperature have been proposed in our recent papers. Simplest applications of these equations to a strain evolution during the multiple temperature cycling in a small temperature interval have been also discussed. Some other problems associated with the irreversible processes caused by hysteresis will be discussed in the present paper. In particular, a new interpretation of DE method based on the transition probability concept and high order approximation including the return point effect is considered.
\end{abstract}

\section{INTRODUCTION}

The main thermomechanical properties of shape-memory alloys are directly connected with martensiic type phase transitions in these systems. Because the martensitic transformations, as a rule, are the first order transitions, a special attention should be attracted to a hysteretic behavior of shape-memory alloys. The most important characteristics of the temperature- or stress-induced martensitic transformations have been studied in detail in [1-5]. It has been shown that such the macroscopic state variables as inelastic strain or volume fraction of the martensite are always complex multi-valued functions of the temperature ard external stress. Therefore, the shape-memory alloys should be considered as systems having an infinite number of state equations that represent the inelastic strain and volume fraction of martensite as functions of the external stress and temperature, correspondingly.

Some of the phenomenological approaches being developed for the thermomechanical state equations of shape memory alloys were recently published in [6-10,14-16]. In particular, a special type of differential equation describing evolution of the inelastic macroscopic strain and volume fraction of martensite as a function of the temperature has been proposed in our recent papers $[11,12,20,21]$. Its application to partial temperature cycling processes in shape-memory alloys and some other problems associated with the irreversible processes caused by hysteresis have been discussed in $[21,22]$.

During the last time the similar models were studied in [17-19]

\section{GENERAL ASPECTS OF MT-KINETICS}

As well known for the martensitic type phase transitions, the temperature and mechanical stress are the main external forces that can essentially influence the transformation process. In particular, as direct as well as reverse transformation process can be induced in the material due to the corresponding temperature or stress change. The main macroscopic state variables such as martensite volume fraction and macroscopic strain represent quantitatively the transformation result. As follows from different experimental studies, all the state variables are very complex multi-valued functions of the external driving forces. Speaking exactly, 
the state variables can not be more considered as functions of current values of the stress or temperature, but instead, they become functions of the external driving force change. The main cause of such a behavior is connected with the thermomechanical hysteresis of the materials undergoing first order diffusionless martensitic phase transition.

In particular, the volume fraction of martensitic phase can be considered as the most representative macroscopic state variable for the temperature-induced martensitic transformation occurring without any external stress applied. In this case, the transformation process can be represented by the main hysteresis loop describing martensite volume fraction change during as direct as well as reverse martersitic transformation on cooling and heating, respectively. Besides of the complete transformation cycle, a set of sub-loops representing partially direct and partially reverse transformation processes are usually observed, as indicated in Fig. 1. Each of partial sub-loops is characterized by a definite return point where, the cooling process is replaced by heating or vise versa.
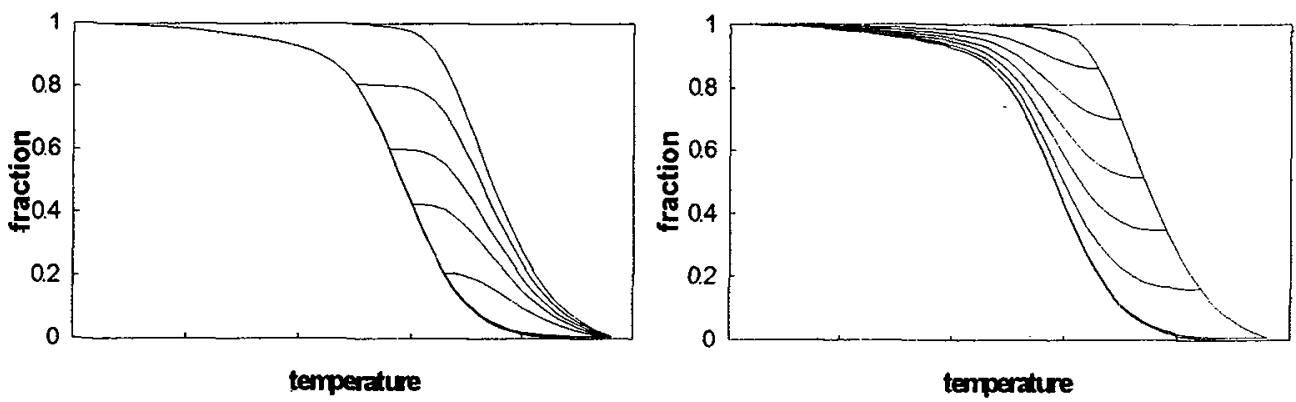

Fig. 1. Typical scheme of the martensite volume fraction behavior during partial MT.

It should be also noted that each point inside the main loop represents one of the possible stationaty two-phase state of the system. All these are the metastable ones, because no volume fraction change is usually observed if one stops the cooling or heating process at this point for any time. This also denotes that thermal fluctuations do not play any significant role in MT. As a result, transitions from one metastable state to another one are only possible on driving the system with any external thermodynamical force, such as a temperature or stress. Therefore, the transformation paths in Fig. 1 represent continuous sequences if metastable states that the system can run on driving by the temperature change process.

Definite efforts have been made by several authors to find the corresponding constitutive methods to describe the main peculiarities of MT-kinetics. In particular, Cory and McNichols [6] have developed the approximation scheme of MT based on an infinite set of state equations representing evidently families of partial transformation trajectories. Likhachev and Koval $[11,12,20,21]$ have proposed a special type differential equation describing evolution of the inelastic strain and volume fraction of martensite during the partial temperature-induced MT. Recently, Ivshin and Pence $[17,18]$ considered other differential model that is similar, but not identical, to that studied in $[11,12]$ and analyzed the nonlinear modifications for both the models [19].

\section{REPRESENTATION OF MT-KINETICS IN FRAMEWORK OF DEM}

As follows from the results represented in Fig. 1, the macroscopic volume fraction of martensitic phase can be characterized by the main hysteresis loop $\chi=z_{-}(T)$ and $\chi=z_{+}(T)$ corresponding to cooling and heating processes, respectively. Furthermore, a set of sub-loops representing partial transformation processes are also possible as shown here. Therefore, the martensite volume fraction is always a definite function of the temperature change process and can take arbitrary values inside the main hysteresis loop.

The possibility to describe the irreversible evolution of macroscopic variables caused by hysteresis in the framework of differential equation method (DEM) follows immediately from the assumption that only a single path from each family in Fig. 1 can pass through a given point with coordinates $(\chi, T)$ inside the main hysteresis loop. Therefore, only a single value of the first derivative $d \chi / d T$ for the cooling or heating 
family of paths may correspond to each point in the $(\chi, T)$ plane. Mathematically, this denotes that $d \chi / d T$ must be a single-valued function of $\chi$ and $T$ separately for cooling and heating processes. Accordingly, each thermodynamical path belonging to the cooling or heating family must satisfy the following type of differential equations:

$$
d \chi / d T=S_{ \pm}(\chi, T)
$$

where $S_{ \pm}(\chi, T)$ are single-valued functions of $(\chi, T)$ associated with the heating and cooling processes that are signed as $( \pm)$, respectively. It has also been found that $S_{ \pm}(\chi, T)$ can be expressed as a linear function of $\chi$

$$
S_{ \pm}(\chi, T)=\alpha_{ \pm}(T) \chi+\beta_{ \pm}(T)
$$

with the temperature dependent coefficients $\alpha_{ \pm}(T)$ and $\beta_{ \pm}(T)$ depending only on the main hysteresis loop shape represented by $z_{ \pm}(T)$ functions:

$$
\alpha_{ \pm}(T)= \pm \frac{d z_{ \pm}}{d T} \frac{1}{z_{+}-z_{-}} ; \quad \quad \beta_{ \pm}(T)=\mp \frac{d z_{ \pm}}{d T} \frac{z_{\mp}}{z_{+}-z_{-}}
$$

Therefore, the differential equations for the basic heating and cooling paths family are:

$$
d \chi / d T= \pm \frac{d z_{ \pm}}{d T}\left(\frac{\chi-z_{\bar{\mp}}}{z_{+}-z_{-}}\right)
$$

These equations make it possible to predict the volume fraction evolution for any temperature change process. In accordance with Eq.(4), for this aim one should know only the information about the temperature behavior of $z_{ \pm}(T)$-paths representing the main hysteresis loop.

These can be also represented as the time-evolution equations of $\chi(T)$. Because the time derivative of $\chi(T)$ is $\dot{\chi}=\dot{T}(d \chi / d T)$ and using Eqns. $(1,2)$ one can easily obtain:

$$
\dot{\chi}=\dot{T}\left(\alpha_{ \pm}(T) \chi+\beta_{ \pm}(T)\right)
$$

This one can be considered as a special kinetic equation describing athermal type of martensitic transformation kinetics.

\section{TRANSITION PROBABILITY CONCEPT}

To understand better the nature of differential equation method a very important physical interpretation based on the master kinetic equation concept proposed in [25] will be considered in this section. As follows from this well known in physics general concept, the kinetic equations describing reactions between two phases $A \Leftrightarrow M$ (austenite $\Leftrightarrow$ martensite) can be represented in the following general form:

$$
\begin{aligned}
& \dot{x}_{m}=-W^{m \rightarrow a} x_{m}+W^{a \rightarrow m} \chi_{a} \\
& \dot{x}_{a}=-W^{a \rightarrow m} \chi_{a}+W^{m \rightarrow a} \chi_{m}
\end{aligned}
$$


These equations represent the temporal evolution of the martensitic (m) or austenitic (a) phase fraction due to the local $m \rightarrow a$ and $a \rightarrow m$ microscopic transformation processes with the corresponding transition probabilities: $W^{m \rightarrow a}$ and $W^{a \rightarrow m}$. Here, $\dot{\chi}_{m}, \dot{\chi}_{a}$ denote the rates of $\chi_{m}, \chi_{a}$ change, correspondingly. The considered kinetic equations satisfy automatically the following conservation rule:

$$
\chi_{m}+\chi_{a}=\text { const }=1
$$

Due to this rule, representing for example the austenitic fraction as $\chi_{a}=1-\chi_{m}$ and substituting it into the first master equation one can easily obtain the single evolutionary kinetic equation for the martensitic phase fraction:

$$
\dot{\chi}_{m}=-\left(W^{m \rightarrow a}+W^{a \rightarrow m}\right) \chi_{m}+W^{a \rightarrow m}
$$

Comparing this equation with Eq.(5) found in framework of DEM one can easily see that those are similar each other. Therefore, the corresponding kinetic coefficients, following as from DEM as well as from master equations, must be equal too. That is, the following relations can be immediately found:

$$
(\dot{T}) \alpha_{ \pm}(T)=-\left(W^{m \rightarrow a}+W^{a \rightarrow m}\right) \quad(\dot{T}) \beta_{ \pm}(T)=\left(W^{a \rightarrow m}\right)
$$

By using the results represented by Eq.(3) the evident expressions for the transition probabilities can be found as follows:

$$
W^{a \rightarrow m}=( \pm \dot{T})\left(-\frac{d z_{ \pm}}{d T}\right)\left(\frac{z_{\mp}}{z_{+}-z_{-}}\right) \quad W^{a \rightarrow m}=( \pm \dot{T})\left(-\frac{d z_{ \pm}}{d T}\right)\left(\frac{1-z_{\mp}}{z_{+}-z_{-}}\right)
$$

It is important that both $W^{a \rightarrow m}$ and $W^{m \rightarrow a}$ are always positively defined functions because all the terms included into the brackets are positive. This denotes that their interpretation as the transition probabilities is physically correct.

\section{CONSTRUCTION OF HIGH ORDER APPROACH}

It is important to note that the possibility to describe the partial transformation processes in the framework of differential equation (1) is essentially based on the assumption that only a single partial trajectory from heating or cooling family can pass through a given point $(\chi, T)$ inside the main loop. This also denotes that the transformation trajectories from heating (or cooling) family cannot intersect each other. As follow from different experimental studies DEM usually represent a good quantitative scheme of calculation for the first order partial sub-loops in real shape-memory materials. In this case the misfit between the experimental and simulated transformation paths remains very small. But, one can observe some more misfit for the second order transformation paths containing a set of the high order internal return points inside the main hysteresis loop [24]. This misfit is localized in the definite temperature intervals nearly to these return points. Therefore, one can conclude that DE-method can give good quantitative results asymptotically in the limit when the partial trajectory will be far enough from the corresponding return point. In this limit the misfit between the model and experiment will disappear. In other case when these requirements cannot be satisfied one should take into account definite pre-history effects. In other words, the current value of temperature slope cannot be more dependent only on the current values of the fraction and temperature, but must be also dependent on the other pre-history variables such as the preceding return points temperatures. 
To take into account similar return point effects we propose to change the main constitutive equation (1) of DEM by the more general version proposed in [23]:

$$
d \chi / d T=S_{ \pm}^{R P F}\left(\chi, T, T_{R}\right)
$$

where the local slope $d \chi / d T$ of transformation path becomes the function dependent as on the current values of $(\chi, T)$ as well as on the last return point temperature $T_{R}$.According to an asymptotic character of $\mathrm{DEM}$ we will require that new functions $S_{ \pm}^{R P F}\left(\chi, T, T_{R}\right)$ approached to corresponding $S_{ \pm}(\chi, T)$-functions found in the framework of DEM in the limit $\left|T-T_{R}\right| \rightarrow \infty$. On the other hand the transformation path slope becomes usually very small nearly the return point temperature. So, one can conclude that $S_{ \pm}^{R P F}\left(\chi, T, T_{R}\right) \rightarrow 0$ if $\left|T-T_{R}\right| \rightarrow 0$. To satisfy these requirements, it is convenient to express $S_{ \pm}^{R P F}\left(\chi, T, T_{R}\right)$ as follows:

$$
S_{ \pm}^{R P F}\left(\chi, T, T_{R}\right)=R\left(T-T_{R}\right) S_{ \pm}(\chi, T)
$$

where the return point effect is represented by the return point factor $R(\tau)$. It is evidently that $R(\tau) \rightarrow 0$ if $|\tau| \rightarrow 0$ and $R(\tau) \rightarrow 1$ if $|\tau| \rightarrow \infty$. In this paper we will use a simple model representation of the return point factor satisfying these conditions:

$$
R(\tau)=1-\exp \left(-|\tau| / \tau_{R}\right)
$$

Here, the material constant $\tau_{R}$ defines the temperature interval $\left|T-T_{R}\right| \leq \tau_{R}$ where the return point effect is the most essential. Inside of this interval $d \chi / d T$ takes very small values different from those predicted by DE-method. But, both methods become equivalent asymptotically, if only $\left|T-T_{R}\right| \geq \tau_{R}$. As a result, the local slope $d \chi / d T$ will not be more a single valued function of current values of $(\chi, T)$ because it can take an infinite set of values between $S_{ \pm}(\chi, T) \leq d \chi / d T \leq 0$ in this new version of DEM. Therefore, one can find an infinite set of partial transformation trajectories intersecting each other at any given point inside the main loop. Comparison between the usual DE-method and its new modification taking into account return point factor (RPF) are represented in Fig. 2.

The possibility to find the intersection points between the partial transformation paths are indicated by arrows in Fig.2.(left). The influence of RPF on the partial temperature cycling behavior is also shown at the bottom side of plots. The main hysteresis loop shape data of Ni-Ti shape-memory alloy necessary to perform the simulation procedure have been taken from [12].
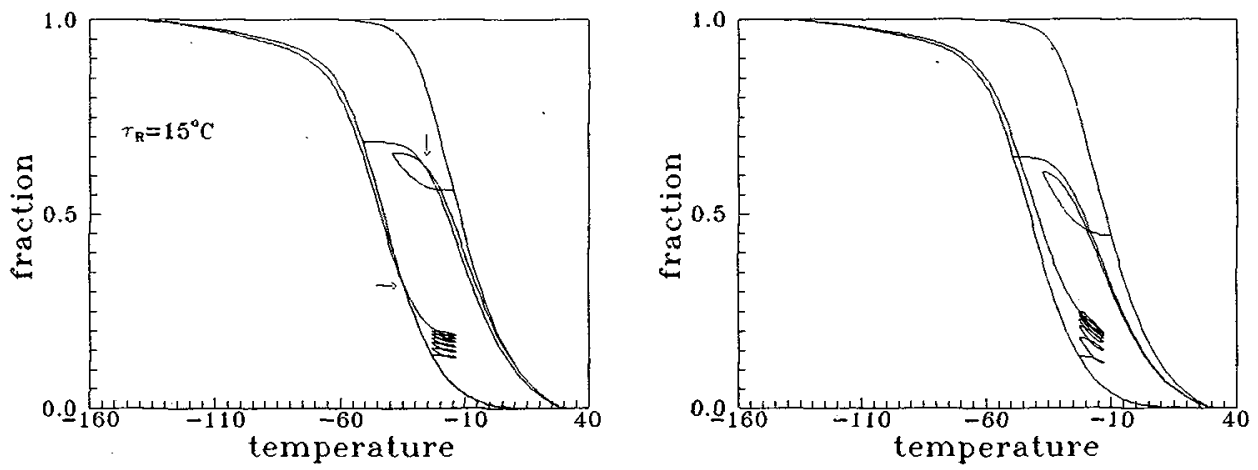

Fig. 2. Return point effects (left) and partial transformation paths simulated according to DE-method (right). 


\section{CONCLUSIONS}

The most important conclusions following from the present study of the athermal type of MT-kineti:s can be formulated as follows.

Successful description of the irreversible evolution of macroscopic variables caused by hysteresis is possible by using a special type of differential equations with the temperature dependent coefficient depending only on the main hysteresis loop shape. These equations make it possible to predict the macroscopic volume fraction evolution for different temperature change process including the partial trajectories.

It is important that the earlier proposed equations can be derived from the well known in physics general concept based on the master kinetic equation if only to apply it to the martensitic type transformation reactions. By using the results found in framework of DEM the evident expressions for the transition probabilities satisfying all the necessary physical conditions can be found too.

The return point effects can be also included into the general scheme of DEM through the return point factor as proposed in the last section of present paper.

\section{Acknowledgments}

This work has been partially supported in the framework of European scientific program INTAS -931202ext.

\section{References}

[1] I.Cornelis and C.M.Wayman., Scr.Met. 10 (1967) 359.

[2] K.Otsuka, C.M.Wayman, K.Nakai, H.Sakamoto, and K.Shimizu, Acta Met. 24 (1979) 207.

[3] L.Delaey, J.Van Humbeeck, M.Chandrasekharan, J.Janssen, M.Andrade, and N.Mwamba Metais Forum. 4 (1981) 164.

[4] Yu.I.Paskal, and L.A.Monasevich., Phys.Met.Metall. 52 (1981) 95.

[5] H.Verguts, L.Delaey, E.Aernoudt, and L.Vermeersch., in EUROUMECH Colloquium, Warshawa, Poland, 1983, p.171.

[6] J.S.Cory and J.L.McNichols., J.Appl.Phys. 58 (1985) 3282.

[7] E.Aernoudt, and Lu Li., in Proceed. Int. Symp on Shape Memory, Guilin, China, 1986, p.23.

[8] L.Delaey, and E.Aernoudt., in Proceed. of ICOMAT-86, Nara, Japan, 1986, p.926.

[9] I.D.Mayergoyz., J.Appl.Phys. 57 (1985) 3803.

[10] P. Guelin, and D.Favier., J. de Mechanique. 19 (1980) 217.

[11] Yu.N.Koval, and A.A.Likhachev., Metallofizika. 5 (1988) 28.

[12] A.A.Likhachev, and Yu.N.Koval., Scr.Met. et Mat. 27 (1992) 223.

[13] J.Van Humbeeck and R. Stalmans., Mat.Sci.Forum. 56 (1990) 405.

[14] J.Ortin., J.Appl.Phys. 71 (1992) 1454.

[15] A.Visintin., "Topics in Nonsmooth Mechanics", J.J.Moreau, P.D.Panagiotopulos and G.Strang, Birkhauser, Basel, 1988.

[16] M.A.Krasnoselÿskii and A.Pokrovskii., "Systems with Hysteresis", Springer,Berlin, 1980.

[17] Y.Ivshin and T.J.Pence., Int.J.Ing.Sci. 32 (1994) 681.

[18] Y.Ivshin and T.J.Pence., Proceed. of ICOMAT-92, July 20-24 1992, (C.M.Wayman and J.Perkiris, eds.), p.389.

[19] R.Loloee, T.J.Pence, D.S.Grummon., Proceed. of ICOMAT-95, Lausanne, 1995.

[20] Yu.N. Koval Yu.N., A.A.. Likhachev., "Martensitic Type Phase Transitions", Kiev, Naukova Durnk:a, 1993, pp. 53-72.

[21] A.A.Likhachev A.A., Proceed. of ESOMAT-94, Barcelona, Spain, Collque C2, Journal de Physique III, 5 (1995) 465.

[22] A.A.Likhachev., Scr.Met et Mat, 32 (1995) 663

[23] A.A. Likhachev and Yu.N. Koval, Metallophysics and Adv. Techn. 17 (1995) 26.

[24] A. Amengual, A.A. Likhachev and E. Cesari., Scr. Met. et Mat. 34 (1996) 722.

[25] A.A. Likhachev and Yu.N. Koval., Metallophysics and Adv. Techn. 18 (1996) 10 